\title{
Using Multiagent Systems and the Internet in Care Services for the Ageing Society
}

\author{
L. M. Camarinha-Matos ${ }^{(1)}$ and W. Vieira ${ }^{(1)(2)}$ \\ ${ }^{(1)}$ New University of Lisbon - Faculty of Sciences and \\ Technology, Quinta da Torre, 2825 Monte Caparica, Portugal, \\ Fax: +351-1-2957786,e-mail: \{cam,wv\}@uninova.pt \\ ${ }^{(2)}$ Instituto Superior de Engenharia de Lisboa, \\ Rua Conselheiro Emidio Navarro, 1900 Lisboa, Portugal
}

\begin{abstract}
One of the most important problems the current society must deal with is the aging of the population, which, along with the increasing engagement of both men and women in work outside their homes, demand new solutions for the care of elderly people. Technology has reached a state where cheap support systems for elderly people staying alone at home can be implemented. A possible solution is to allow care and health centers to remotely observe and help old people staying at their homes. In this paper we present an architecture for the support of elderly people in their daily tasks. Such an architecture is based on the Internet as the communication infrastructure and extensively exploits the Multi Agent Systems framework including both stationary and mobile agents.
\end{abstract}

\section{Keywords \\ Mobile Agents, Multi Agent Systems, Elderly Care Systems}

\section{INTRODUCTION}

\subsection{Motivation}

Elderly people are becoming a very important part of the population of the industrialized countries (Saranummi, 1996), specially in the EU and the USA. 
Some of these people often require special attention and vigilance due to the reduced degree of autonomy they might present.

The traditional approach for taking care of these people has been either resorting to support from relatives or elderly care centers. However these solutions become inappropriate mainly for the following reasons: i) the involvement of relatives is more and more difficult since the social evolution in the developed countries has led to more and more people (specially women) to get involved in work outside their homes; ii) care centers present both cost problems and social uproot of the involved people; iii) many elderly people preserve enough robustness to be at their homes which is very often the solution they prefer and is better for their health.

Technology development has reached a state where we can envisage a solution which allow those elderly people whose degree of autonomy, although reduced, is significant to stay at their homes, provided adequate vigilance services exist.

The degree of vigilance is dependent on the degree of autonomy of the person involved and can be as simple as an active advice and agenda system or it can include more sophisticated equipment (sensors, robotized home appliances, etc.). Yet another very important factor related to elderly is the need for leisure support, since they remain alone at home for long periods of time. This kind of support must include both effective help in the use of home equipment (video, TV, etc.) and services which allow elderly people to find, buy or rent some entertainment materials or to agree on some common schedules with other people in the same situation (a bridge game at some elderly social center, for instance).

The Internet seems to be the best choice for the communication infrastructure for this kind of system due to its low cost and widespread availability. On the other hand, Multi Agent Systems (MAS) (Wooldridge, 1995) have proved to be a powerful framework for the development of complex distributed systems. Also, Mobile Agents (MA) (Chess, 1995) (Camarinha-Matos, 1997) are specially well suited to the development of remote applications over the Internet.

In this paper we describe an architecture intended to help families to maintain their older members at home till longer ages. It is based on the Internet and exploits the MAS and MA paradigms.

\subsection{Plan of the Paper}

The remainder of this paper is structured as follows. In section 2, a scenario for a hypothetical elderly support system is described in general terms and desired characteristics are pointed out independently of any particular implementation. Section 3 presents a brief description of multi-agent systems and mobile agents. Section 4 is dedicated to the presentation of an architecture for such a system. In section 5, the implementation of some subsets of this architecture is discussed. Finally, in section 6, current limitations and future work are addressed.

\section{NEEDS OF ELDERLY SUPPORT SYSTEMS}

In this section we describe a scenario for a hypothetical elderly support system. The main requirements for such a system are pointed out. 
The elderly needs. At the base of the system we have the elderly people who, in spite of their natural loss of capabilities, maintain enough autonomy to, securely and adequately, carry out their daily tasks if appropriate support is given them. These people spend most of their time at home but, occasionally, may go out for short periods of time with several possible destinations: heath centers, social care centers, elderly day centers, leisure centers, etc.. This people often exhibit some medication and alimentary care needs and are susceptible to sudden changes in their health state.

In order to provide the appropriate level of support for these people, it is, therefore, necessary to have some vigilance and advice/help systems located at their homes and at the places they visit (elderly care centers, health centers, etc.). Additionally, some form of personal information maintenance, which includes medication an food constraints, personal interests and preferences, etc. must be provided.

Home sites. A form of guaranteeing security of elderly people when staying at home and help them in their daily tasks (use of home appliances, for example) must be found. Also, elderly people must be engaged in leisure activities in order to not feel lonely. Therefore some way to propose leisure activities (video, music, etc.) and to help elderly persons in the use of home equipment must be provided. It should be noted that this is a highly heterogeneous scenario: each home environment and each person needs are distinct.

Care centers' needs. Nowadays, social care centers face big difficulties since they must either employ a lot of social assistants or choose to rend their services within the walls. In order to encompass these limitations a system offering remote social care services is needed. Services for helping/reminding elderly persons at home and monitor their activity from the social centers must be provided.

Health centers' needs. Elderly people often require special health vigilance, which includes monitoring of their physical and emotional conditions and the fulfillment of some medication agenda. Since the available resources at the health centers are very scarce, it is often the case that one doctor or nurse have many elderly people to take care of, which frequently implies poor service quality. So, it is necessary to find some kind of remote monitoring/vigilance of the elderly persons' health sate, in order to improve the quality of the offered services. The results achieved can also be used for remote health care of other patients (Miksch, 1996).

Relatives' needs. As previously mentioned, in the developed countries it is very common that the elderly person's relatives are engaged in jobs outside their homes. Consequently, they cannot offer personal care during their absence. However, in general, relatives can and want to offer help/advice to their elderly persons. Moreover, relatives will feel better themselves if they have some way to observe the activity and help the elderly persons from their jobs. A remote monitoring/vigilance facility is, therefore, an important functionality that could be operated from the job site.

Leisure centers' needs. Leisure centers are extremely important for the elderly people in what concerns their free time occupation. In fact, day centers provide 
support for a user to buy or rent some materials (books, videos, meals, etc.) and allow users to get engaged, either locally or remotely, on several social activities, such as a bridge game with other users, for example. The limitations, needs and preferences of the elderly persons must somehow be considered in these centers and supported by technology.

Sales services. Due to the reduced mobility of elderly people, some sort of advertisement/sale of products must be provided which allow an easy way for these people to choose and buy some goods. These services can exist in a leisure center or in specialized centers for this purpose. The rapid developments in the Electronic Commerce area have to be analyzed in terms of the specificity of this social sector.

Safety and privacy issues. One of the most critical aspects to be considered in this kind of support system is the users (elderly persons) safety. So, special effort must be put in providing local and remote vigilance agents which must preserve the users safety with a very high degree of certainty. Not only shall the sensors data be analyzed but also the users activity must be monitored (using presence sensors, image sensors, etc.). Depending on the health situation of the user, his/her personal data must be updated on the remote center at a sufficiently high rate in order to allow adequate response in case of failure of the home equipment or loss of communication between the remote center and the user home. If appropriate, redundancy must be implemented for the critical systems.

However, there is a trade-off between users' safety and privacy. Since a system which doesn't preserve enough privacy will not be attractive to the users, a balanced approach have to be found. Although the degree of privacy to be preserved depends on the health situation of the user, there are lower bound limits that must be guaranteed.

\section{MULTI-AGENT SYSTEMS AND MOBILE AGENTS}

Multi-agent (intelligent) systems (MAS) are software systems composed of several autonomous software agents running in a distributed environment. Besides the local goals of each agent, global objectives are established which commit all or some group of agents to their completion. Some advantages of this approach are: i) it is a natural way for controlling the complexity of large highly distributed systems; ii) it allows the construction of scalable systems since the addition of more agents is an easy task; iii) multi-agent systems are potentially more robust and tolerant to failures than centralised systems.

Coordination of the agents is a crucial aspect of multi-agent systems. Several coordination approaches have been suggested ranging from pure organisational to social inspired, or those based on market theories as is the case of the contract net protocol (Smith 1988).

Mobile agents have also gained more and more attention of researchers in the last years. Essentially, a mobile agent has the ability to decide to move from one machine to another. When this happens the execution state of the agent is saved 
and moved along with its code to the destination machine where the execution of the agent will be resumed.

Several advantages of mobile agents have been pointed out: i) they may reduce bandwidth requirements, since in moving to the machine where the data is, they can perform all the computation there and transmit just the result to the original machine; ii) they can act autonomously and even in the absence of a network connection to the machine from where they were launched (an important aspect, for example, in mobile computing); iii) they provide high scalability to the systems since functionality may be added whenever needed; iv) for remote operation, they show significant advantages, because they conciliate flexibility and reliability (Camarinha-Matos, 1997), since in moving to the place where the machines or devices to be operated are, they provide execution supervision locally, and so, they do not rely on the availability, delays and reliability of the network.

However, mobile agents present some difficult problems: i) they require special servers to run; ii) security is a major concern in mobile agents. In fact, "how can servers trust mobile agents", and "how can we protect agents from servers" are hot research domains.

Both MAS and mobile agents suffer from the lack of standardisation which has been an obstacle for a faster development of these technologies.

\section{PROPOSED ARCHITECTURE}

Many of the characteristics referred to in section 2 can be supported by current technology. Some of them require additional progress, as is the case of home appliances which, besides automated operation capabilities, must integrate some kind of remote control (from a personal computer, for example). The authors think that the evolution in this direction is just a matter of time.

As this system reveals high geographical distribution, Internet is a reasonable choice due to its low cost and widespread availability. Using the Internet, homes, care centers, health centers, and leisure centers can be inexpensively linked despite the distances among them.

For the monitoring of the health state of the elderly persons, as well as for the observation of their activity, a lot of sensors and measurement devices exist, ranging from very simple presence sensors to sophisticated blood pressure measurements devices. Therefore it is possible to implement intelligent monitoring systems either for the vigilance of the elderly persons' health states and the observation of their daily activity at their homes.

It is almost certain that future developments in home appliances will allow them to be interconnected in a local network and to be controlled from a computer. This will make it possible to implement sophisticated help systems for elderly people. In particular, the use of image and voice sensors in conjunction with such sort of intelligent home appliances allows the implementation of intelligent adaptive interfaces for helping/guiding elderly persons in their daily tasks.

On the other hand, developments in the area of multi agent systems have proved to be very effective in the development of complex distributed systems such as the 
one we are discussing. Furthermore, mobile agents are recognized to be very appropriate to the development of distributed applications over the Internet, due to their low bandwidth requirements and flexibility/scalability characteristics. In addition, developments in the field of the smart cards, cellular phones and handheld network communicators will allow the implementation of personal mobile agents which "live" inside such devices and which accompany the user wherever he/she goes, carrying the user's personal history and data. Therefore, it is the authors' opinion that a powerful, cheap and flexible system can be realized in the near future.

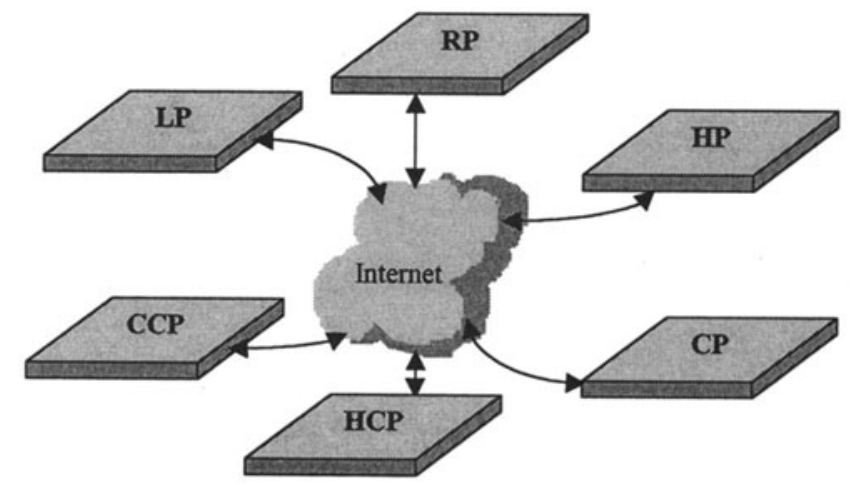

Figure 1 - Several types of places interconnected through the Internet

According to the statements made above, a multi-agent architecture based on the Internet and which exploits the mobile agents paradigm is proposed.

As shown in Figure 1 the general framework of the proposed system includes several places where elderly persons live ("Home Places" - HPs), care center places ("Care Center Places" CCPs), health center places ("Health Center Places" HCPs), several leisure places ("Leisure Places" - LPs) and several places from where relatives may observe the behaviour of the elderly people staying in a HP or give him/her some help ("relatives' places" - RPs). Places for the advertisement/buying of goods are also considered ("commerce places" - CPs).

A HP is a place where an elderly person lives and stays alone for long periods of time. So, it must have a convenient environment in order to appropriately implement the characteristics discussed in section 2. Figure 2 a) represents a structure for a HP. As it shows, each HP may comprise a set of sensors (temperature, presence, etc.), a set of measurement devices (blood pressure, body temperature, etc.) and a set of robotized home equipment (microwave ovens, air conditioning equipment, light control equipment, etc.). The main components of a $\mathrm{HP}$ are: i) the monitor agent (HP-MA) which is responsible for the vigilance of the welfare of the elderly person, by analysis of sensor values (environmental and physical measures); ii) the agenda agent (HP-AA) which is responsible for guaranteeing the fulfillment of a pre defined agenda (for example, for taking care of the time when some medicines have to be taken by the elderly person); iii) the advice/help agent (HP-HA) which, by means of intelligent adaptive interfaces, is 
responsible to give the elderly person advice or help in several occasions (how to use a HIFI equipment, for example); iv) the mobile agents support system which allows mobile agents sent from care centers, health centers or from a relative's job to run in the HP (HP-MAS). The HP-MAS also allows a HP to launch mobile agents (for example, a mobile agent can be launched to go visit some $\mathrm{CP}$ in order to buy some goods); v) an interface with the elderly person's personal agent (HPPAI). The personal agent can reside in a smart card, or in a cellular phone, for example.

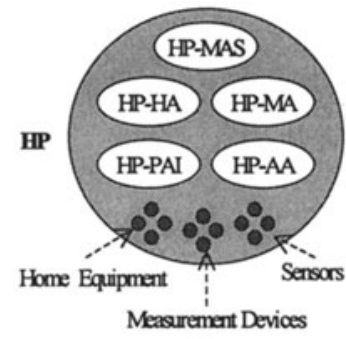

a)

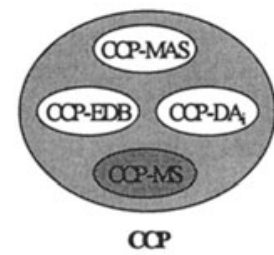

b)

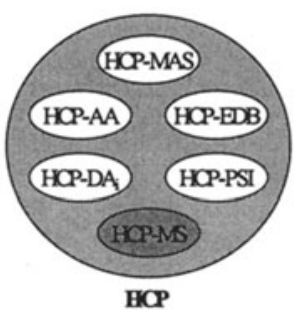

c)

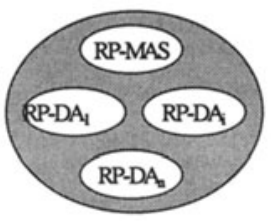

RP

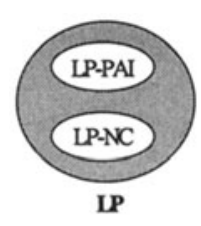

d)

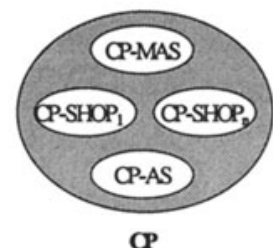

e)

f)

Figure 2 - Structure of HPs, CCPs, HCPs, LPs, CPs and RPs

In alternative, the HP-MA and HP-AA functionalities would be performed by mobile agents sent by HCPs, CCPs, or RPs.

A CCP allows institutions which provide care to elderly to do that in a remote manner, whenever the degree of autonomy of the elderly person allows him/her to stay at home. As shown in figure $2 \mathrm{~b}$ ), a CCP may launch mobile agents to run in some HP (using the mobile agents support, CCP-MAS). These agents are intended to give some kind of support/help to the elderly person. For example, a mobile agent can be sent to help the elderly person in food preparation. The adoption of mobile agents for the services a CCP can provide, allows a high degree of flexibility and scalability for the system. A CCP also includes a database with elderly persons' data (CCP-EDB) and a management system (CCP-MS), which is responsible for the global management of the CCP (addition of a new user, removing of a user, launching agents to the HPs, etc.).

A HCP allows remote rendering of health services and vigilance. Again this is possible only in those cases where the elderly persons preserve enough degree of 
autonomy. As figure 2 c) shows, a HCP is composed of the following systems: i) the alarm agent (HCP-AA) which is responsible for the treatment of the alarms received from monitor agents in the home places (HP-MA); ii) support to launch mobile agents (HCP-MAS) to run in some HP for some kinds of health care or health vigilance; iii) an interface to the users' (elderly people) personal agents (HCP-PAI) (for updating their data when they visit the health center) ; iv) a database with users' data and a management system also exist in a HCP.

A LP is a place which the elderly person can visit and where he/she can eat some meal, rent some book or get engaged in some social activity with other elderly persons. A LP contains an interface with the elderly persons' personal agents (LPPAI) in order to acquire the users preferences and food and medication restrictions. A LP also provides some form of negotiation/coordination between personal agents (LP-NC). This enables the personal agents of two or more elderly persons to negotiate the engagement of their owners in some common social activity. Figure 2 d) represents the structure of a LP.

A commerce place (CP) is a virtual market where companies can advertise and sell goods. It comprises a set of agents which represent some shops (CP-SHOPi). Mobile agents specialized in shopping can be sent from a HP to buy some goods in a CP, so, support for execution of mobile agents must be provided (CP-MAS). Also, support for advertisement (CP-AS) must be included in a CP which allows shopping mobile agents to search the advertisement lists and find the products they search. A CP can be integrated in a LP. Figure 2 e) represents a CP.

Finally, a RP is a place from where a relative may send a mobile agent to a HP for observing or giving some help to the elderly person who lives there. A RP comprises the support for launching mobile agents (RP-MAS) and agents that support the dialog with the agents sent to a HP (RP-DAs), as figure $2 \mathrm{f}$ ) shows.

Besides the places discussed above, a large sort of mobile agents can exist within this architecture. The following are some of them:

- A care center place may send several mobile agents to a home place. For example, agents to help elderly persons in food preparation, to monitor their activity, etc..

- A health center place may send mobile agents to a home place for observing some specific conditions of the elderly persons (measure his/her blood pressure, for example).

- From RPs, relatives may send mobile agents to a home place for helping/ reminding or for observing the elderly persons' behaviour.

- A home place may send a mobile agent to visit some commerce places in order to buy some goods.

The mobile agents sent to a HP from RPs, CCPs or HCPs must have some capabilities to adapt themselves to the exact environment they reach, since, depending on a number of factors (economic situation, or level of disability, for instance), different HPs will have different capabilities. Therefore, some mechanism must be provided to allow this kind of self-adaptation. 
Finally, personal agents, which act as representatives for elderly persons, must be guaranteed to be updated with the most recent personal data and history of their owners.

\section{IMPLEMENTATION}

The implementation of all the aspects involved in the architecture described in section 4 is a huge task. So a subset of those aspects was chosen for a prototype implementation. This subset comprises the support for launching and running mobile agents, and most of the aspects involved in a HP. The implemented part already allows mobile agents to be sent by CCPs and HCPs for execution in a HP.

Since the described architecture is highly distributed and its main components may run on different platforms, a platform-independent language had to be chosen. Java (Golsling, 1995) was chosen because it is platform-independent and it already provides some mechanisms for remote downloading of code and for security implementation. Additionally, JESS ("Java Expert System Shell") (Friedman-Hill, 1997) was integrated in the system. JESS is a clone of the well known rule based expert system shell CLIPS (Giarratano, 1993) for running in Java. By using JESS it is possible to develop modules in a symbolic rule based language which provides more flexibility an eases the rapid development of complex systems.

\subsection{The Monitor Agent}

Within a HP the monitor agent is responsible to observe the welfare of the elderly person. By reading some sensors it can decide about the environmental conditions as well as the physical conditions of the person. It can react to some unwanted situation, either by trying to correct the situation (altering the setup of an air conditioning device, for example), or by sending alarms to a health center and giving advice to the elderly person in order he/she can him/herself correct the anomaly.

The implemented system allows the development of the monitor agent either entirely in Java (Camarinha-Matos, 1997), or using Java and Jess. Figure 3 a) shows how rules can be defined in Java and figure $3 \mathrm{~b}$ ) shows how equivalent rules would be defined in JESS.

\subsection{The Agenda Agent}

The agenda agent runs within a HP and is responsible for guaranteeing the fulfillment of some pre-defined agenda. The agenda may contain a medication time-table, a meal time-table, etc.. Mobile agents sent by HCPs, CCPs or RPs may modify the agenda. The agenda agent works essentially in a time-driven basis. Mobile agents, sent from CCPs, HCPs or RPs, may set some entries in the agenda.

Again, this kind of agent can be implemented using Java only, or using Jess and Java. 


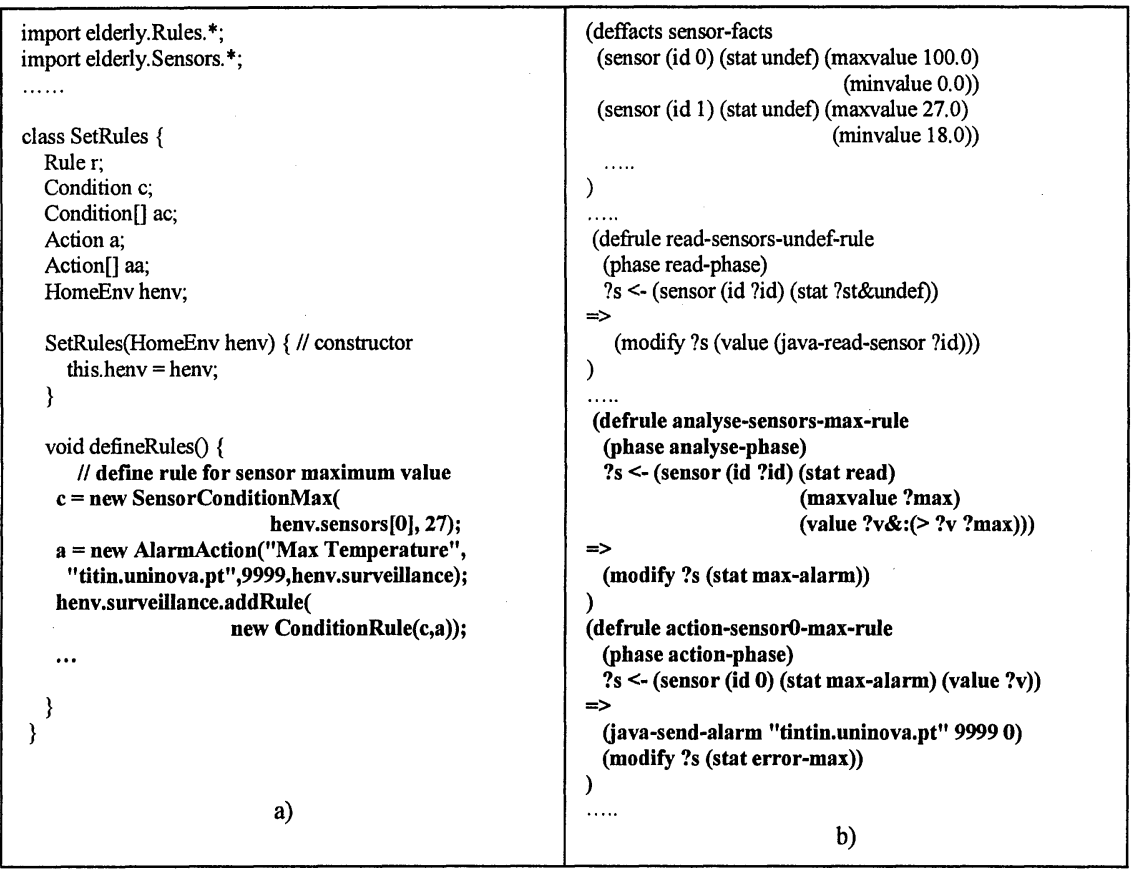

Figure 3 - Definition of rules for a HP-MA: a) using Java, b) using Jess

\subsection{Mobile Agents Support}

A system (IMAJ - "Inceptive Mobile Agents is Java") (Camarinha-Matos, 1997) which supports launching and running of mobile agents was developed. This system allows the creation of Java mobile agents which run on specialized servers. Either the construction of the mobile agents and the building of the servers are supported by the system. Figure 4 shows how a system based on IMAJ works.

Clients launch mobile agents for execution in some server (arrow marked launch in figure 4). Once a server receives an agent (arrows marked receive in figure 4) it creates an execution thread for control of the agent execution (circles marked Agent $_{i}$ in figure 4). The agents will run within the environment of the server which imposes several restrictions to the agent. For example, an agent cannot do anything that uses the local environment in an unauthorized way. All it can do must be done by using the services provided by the server (circle marked Services in figure 4). Services are provided for access to local resources as well as for sending a mobile agent to another server when the agent requests it (arrows marked send in figure 4).

As for the monitor and agenda agents within a HP, they may be totally built in Java or may include some source code in Jess. As figure 5 shows, support is given to allow the agents to carry Jess code with them when they travel.

Line 10 shows how a JessCode object can be used to carry Jess code (the contents of the file "Agent.clp)" whereas lines 19 and 20 show how the agent executes the code whenever it reaches a server. Support is given to interface Jess 
code with the agent's Java code. In particular, Jess code can set and read the array of objects named param in lines 3 and 20.

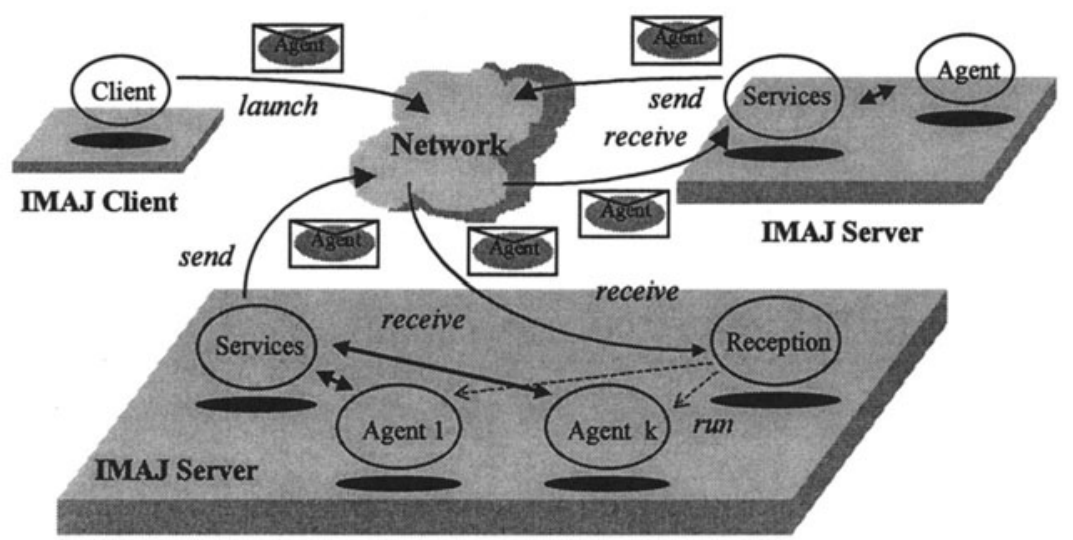

Figure 4-IMAJ system for mobile agents in Java

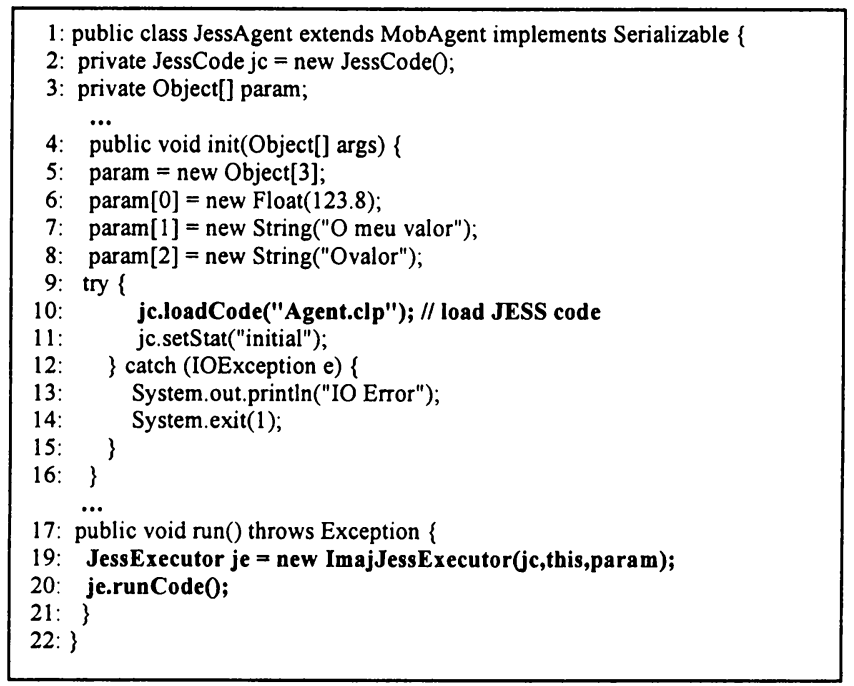

Figure 5 - How mobile agents can carry Jess code

As previously mentioned, one essential characteristic of a mobile agent is its capability to adapt itself to the environments it finds in the places it visits. The possibility of an agent to carry Jess code simplifies the programming effort necessary to accomplish this objective. Currently, a simplified process is implemented which works as follows:

A mobile agent carries with it a general partial order plan for the execution of its tasks, along with several alternative sub-plans for each of the actions in the general 
plan. Each of these sub-plans is associated to some set of local capabilities. When the agent arrives at a server, it requests the list of the local capabilities and, based on this list, it refines the general plan producing a plan suitable for execution (currently, only sequential execution is provided). Of course, a common taxonomy for the description of the capabilities is used. Figure 6 illustrates this process.

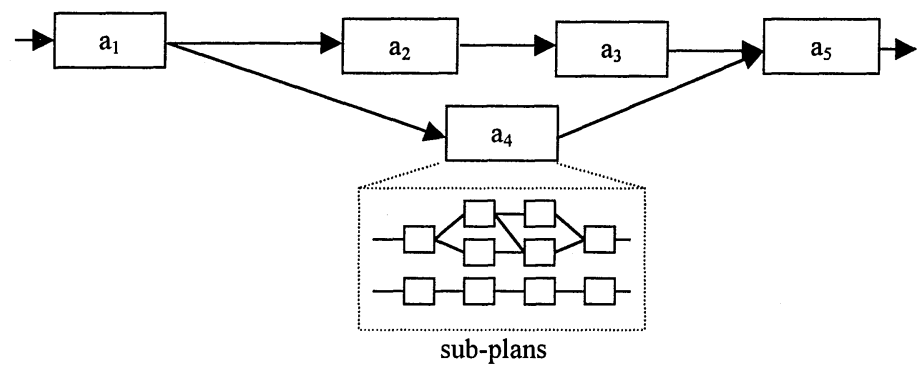

Figure 6 - Illustration of the refinement of general plans

As an example, let's consider HPs where the following equipment may exist:

- To inform the user, either a synthesized voice equipment (voice capability) or a beep with programmable frequency (beep capability) exist;

- Either a robotized microwave (rob-microwave capability) or a gas-stove (gasstove capability) exist for warming up meals.

- A presence sensor exists in the kitchen (presence capability).

Let's imagine a mobile agent sent from a RP to help the user warming up some food for his/her lunch. Such an agent could carry a general plan like the one shown in figure 7.

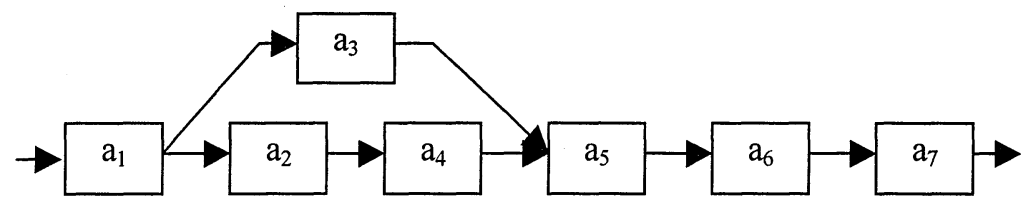

\section{Legend}

$a_{1}-$ call user's attention; $a_{2}$ - get meal from refrigerator;

$a_{3}$ - set the table; $a_{4}$ - warm the meal; $a_{5}$ - serve the meal

$a_{6}$ - wait till eating finished; $a_{7}-$ clear the table

Figure 7 - General plan for helping user in meal warming up

For each of the actions $a_{1}-a_{7}$ there are several possible sub-plans whose application depends on the available capabilities. For example, action $a_{4}$ may have 
two alternative sub-plans, one for a robotized microwave and another for a conventional microwave. The former sub-plan would specify the sequential execution of low level actions such as "open the microwave oven door", "wait until the door is closed", "setup the microwave for 1.5 minutes", "start the microwave" and "wait until done". The last sub-plan could specify a series of indications (using either the voice or beep capabilities) in order to guide the user in the task of setting up and starting the microwave.

For the adaptation of the global plans carried by the agent, a taxonomy was defined for the capabilities each site may have. When refining a global plan, a subplan is built for each of the global actions. In this process, the local environment is requested to describe its capabilities (by a set of Jess facts). For example, if a local site has a robotized microwave oven in the kitchen, the Jess fact (available-cap rob-microwave local-id) is asserted, where rob-microwave is the classification of the capability according to the defined taxonomy and local-id is a local identifier of the device which must be used whenever an operation is to be executed on the device (through activation of specialised Java functions). The attributes for the class rob-microwave include the definition of the possible operations for this class (for example, (capability rob-microwave (open-door 0) (setup 1) (wait-close 2) (start 3) (wait-done 4)) indicates that the capabilities of this class have four operations whose codes for local activation are the integers indicated in each pair). For each global action a sub-plan is generated according to these descriptions of local capabilities.

A global plan is represented by a set of Jess facts indicating for each step its precedence dependencies and its action (according to a defined taxonomy). Two possibilities exist for the generation of sub-plans. The simplest one uses the description of local capabilities to find the sub-plan in a library of sub-plans carried by the agent. The second alternative involves a simple STRIPS-like planner using operator definitions carried by the agent, whose pre-conditions are based on the existing capabilities. The first alternative is well suited when the number of different capabilities that may be used for the same global action is small, whereas the second is more flexible and recommended when that number is moderate or large. At the time of this writing only the first possibility is implemented.

In the current implementation, independence between the sub-plans has been assumed to follow the dependency constraints of the global actions. In other words, it has been assumed that the sub-plans of two unordered global actions can be built independently, which is not true in general.

Figure 8 shows some excerpts of Jess code for the definition of global plans and the simple scheme of plan refining currently in use, based on a library of sub-plans. The Jess fact (action-plan (action-id action) (capab-needs list-of-cap) (sp-id subplan-id)) indicate that sub-plan identified by sub-plan-id may be used for the execution of the global action action, provided the local site has the capabilities indicated in the list list-of-cap. In the excerpts shown in figure 8, action warm_meal of the global plan has two alternative su-plans ( $s p$-id1 and $s p$-id2). The first one is selected if a robotized microwave exists in the local site; otherwise, if a normal microwave and a voice capability exist, the second sub-plan is executed. 


\begin{tabular}{|c|c|}
\hline $\begin{array}{l}\text {; General plan definition } \\
\text { (deffacts plan-facts } \\
\text { (plan-node (id 1) (action call_user_att) } \\
\text { (pred empty) (pred-done empty)) } \\
\text { (plan-node (id 2) (action get_meal_fr_refr) } \\
\text { (pred 1 empty) (pred-done empty)) } \\
\text { (plan-node (id 3) (action set_table) } \\
\text { (pred 1 empty) (pred-done empty)) } \\
\text { (plan-node (id 4) (action warm_meal) } \\
\text { (pred 2 empty) (pred-done empty)) } \\
\text { (plan-node (id 5) (action serve_meal) } \\
\text { (pred 3 4 empty) (pred-done empty)) } \\
\text { (plan-node (id 6) (action wait_eat_finish) } \\
\text { (pred 5 empty) (pred-done empty)) } \\
\text { (plan-node (id 7) (action clr_table) } \\
\text { (pred 6 empty) (pred-done empty)) } \\
\text { ) } \\
\text { deffacts sp-action-warm_meal } \\
\text {; the 2 alternative sub-plans } \\
\text { (action-plan (action-id warm_meal) } \\
\text { (capab-needs rob-microwave)(sp-id 1)) } \\
\text { (action-plan (action-id warm_meal) } \\
\text { (capab-needs normal-microwave voice) } \\
\text { (sp-id 2)) } \\
\text {; the sub-plane 1 (sp-id1) } \\
\text { (sub-plan-node (action warm_meal) (sp-id 1) } \\
\text { (node 1) } \\
\text { (pred empty) (pred-done empty) } \\
\text { (cap-code rob-microwave) } \\
\text { (action-code open-door)) }\end{array}$ & $\begin{array}{l}\text { (sub-plan-node (action warm_meal) (sp-id 1) } \\
\text { (node 2) } \\
\text { (pred 1 empty) (pred-done empty) } \\
\text { (cap-code rob-microwave) } \\
\text { (action-code wait-close)) } \\
\text { (node 3) } \\
\text { (pred 2 empty) (pred-done empty) } \\
\text { (cap-code rob-microwave) } \\
\text { (action-code setup) (parameters 1.5)) } \\
\text { (node 4) } \\
\text { (pred 3 empty) (pred-done empty) } \\
\text { (cap-code rob-microwave) } \\
\text { (action-code start)) } \\
\text { (sub-plan-node (action warm_meal) (sp-id 1) } \\
\text { (node 5) } \\
\text { (pred 4 empty) (pred-done empty) } \\
\text { (cap-code rob-microwave) } \\
\text { (action-code wait-done)) } \\
\text { (sub-plan-node (action warm_meal) (sp-id 1) } \\
\text { (s) } \\
\text {; the sub-plan 2 (sp-id2) } \\
\text { (sub-plan-node (action warm_meal) (sp-id 2) } \\
\text { (node 1) } \\
\text { (pred empty) (pred-done empty) } \\
\text { (cap-code voice) } \\
\text { (action-code setup) } \\
\text { (parameters } \\
\text { "Open the microwave and insert the food")) }\end{array}$ \\
\hline
\end{tabular}

Figure 8 - Excerpts of Jess code for definition of plans

\section{CONCLUSIONS AND FURTHER WORK}

The described scenario for helping elderly people staying alone at home and the problems it carries are of great social impact and, so, require new solutions. The proposed architecture allows an adequate solution for elderly care, either in terms of associated costs and in terms of service quality. The feasibility of this idea has been confirmed by the implemented prototype. However, it is the opinion of the authors that extra effort has to be put on the development of home equipment specialised for this kind of application.

Since the proposed architecture involves a large set of know-how on several domains, only a restricted subset has been implemented in the current prototype. Further work has to be done in several directions: on the implementation of security mechanisms for the mobile agents system, on the improvement of the adaptability part of the mobile agents, on the definition of communication protocols between agents, on cooperation/negotiation between agents, on the aspects related to the user's privacy preservation and on the techniques for information modeling and management in these complex domains. 


\section{REFERENCES}

Saranummi, N., Kivisaari, S., Sarkikoski, T. and Graafmans, J. (1996) Ageing \& Technology. Prepared for the Institute for Prospective Technological Studies European Commission - JRC.

Wooldridge, M. and Jennings, N. R. (1995) Agent Theories, Architectures, and Languages: A Survey. in Intelligent Agents, Lecture Notes in Artificial Intelligence. Springer-Verlag, pp. 1-39.

Chess, D. M., Harrison, C. G. and Kerschenbaun, A. (1995) Mobile Agents: Are they a good idea? IBM Research Report, RC 19887.

Camarinha-Matos, L.M. and Vieira, W. (1997) Mobile Agents and Remote Operation. in Proceedings of the IEEE International Conference on Intelligent Engineering Systems, INES'97, Hungary, pp. 463-468.

Friedman-Hill, E. (1997) Jess, The Java Expert System Shell. URL: http://herzberg.ca.sandia.gov/jess.

Giarratano, J. C. (1993) CLIPS User's Guide, version 6.0. NASA Lyndon B. Johnson Space Center. Information Systems Directorate. Software Technology Branch.

Golsling, J. and McGilton, H. (1995) The Java Language Environment: a white paper. Sun Microsystems, Inc., 2550 Garcia Avenue, Mountain View, California 94043-1100, USA.

Miksch, S., Cheng, K. and Hayes-Roth, B. (1996) An Intelligent Assistant for Patient Health Care. Report No. KSL 96-19. Knowledge Systems Laboratory. Department of Computer Science. Stanford University, Stanford, California.

Yang, Q. (1997) Intelligent Planning: A Decomposition and Abstraction Based Approach. Springer-Verlag, Berlin Heidelberg

Smith, R. G. (1988) The Contract Net Protocol: High Level Communication and Control in Readings in Distributed Artificial Intelligence (ed. A. H. Bond and L. Gasser). pp. 357-366. Morgan Kaufmann

\section{BIOGRAPHIES}

Luís M. Camarinha-Matos is associate professor at the New University of Lisbon where he coordinates the Robotics and CIM group. He has been involved, both as researcher and has technical coordinator, on several international research projects in the areas of virtual enterprises, multiagent systems, intelligent manufacturing systems and machine learning. He has served in the Program Committee of many conferences and was one of the founders of the BASYS conferences series.

Walter Vieira is a $\mathrm{PhD}$ candidate in Electrical Engineering department of the New University of Lisbon (UNL), He graduated from the Computer Science department of UNL in 1981 and received the MSc degree from Instituto Superior Técnico (IST) in 1989. He is an adjunct professor at Instituto Superior de Engenharia de Lisboa (ISEL). His main interests are: distributed intelligent systems and intelligent supervision. 